\title{
Modelo de automatización de procesos para un sistema de gestión a partir de un esquema de documentación basado en Business Process Management (BPM)*
}

Process Automation Model for a Management System Using a Documentation Scheme Based on Business Process Management (BPM)

Modelo de automatização de processos para um sistema de gestão a partir de um esquema de documentação baseado em Business Process Management (BPM)

\section{Karen López Supelano ${ }^{* *}$}

Fecha de recibido: 23 de octubre de 2014

Fecha de aprobado: 26 de marzo de 2015

Doi: dx.doi.org/10.12804/rev.univ.empresa.29.2015.06

Para citar este artículo: López Supelano, K. (2015). Modelo de automatización de procesos para un sistema de gestión a partir de un esquema de documentación basado en Business Process Management (BPM). Universidad \& Empresa, 1729), 131-155. Doi: dx.doi.org/10.12804/rev.univ.empresa.29.2015.06

Este artículo es resultado del proyecto de investigación titulado: "Modelo de automatización de procesos para un sistema de gestión a partir de un esquema de documentación basado en Business Process Management (BPM)" que fue financiado por la Escuela Colombiana de Ingeniería Julio Garavito ubicada en Bogotá (Colombia). El objetivo general de este proyecto es "construir un modelo de documentación para automatizar procesos de un sistema de gestión a partir de un modelo de documentación basado en $B P M{ }^{\prime}$ '.

** Magíster en Ingeniería Industrial con profundización en Sistemas de Gestión Integrada de la Escuela Colombiana de Ingeniería Julio Garavito (Bogotá, Colombia). Directora de la Unidad de Ingeniería de Procesos de la Escuela Colombiana de Ingeniería Julio Garavito (Bogotá, Colombia). Correo electrónico: karen.lopezs@escuelaing.edu.co 


\section{RESUMEN}

Esta investigación pretendió construir un modelo automatizable de documentación de procesos. Se espera que este permita dar respuesta a los requisitos de varios sistemas de gestión, de manera que una organización pueda estandarizarlos y automatizarlos con la confianza de cumplir, al mismo tiempo, con lo establecido tanto por el Consejo Nacional de Acreditación (CNA), como por la norma ISO 9001 y la Business Process Management (вPM). Se analizaron de manera minuciosa y comparativa estos modelos. Los resultados se utilizaron para complementar el contenido del manual. El modelo resultante se aplicó directamente en el contexto organizacional. Los resultados muestran que es posible documentar procesos al detalle, cumplir con estándares internacionales, responder con rapidez a cambios con base en las mejoras que los procesos pueden presentar y llevar el contenido del modelo propuesto a una Business Process Management Suite (BPMS), ahorrando con esto costos, tiempo y otros elementos valiosos.

Palabras clave: Automatización, BPM, documentación, procesos, sistema de gestión.

\section{ABSTRACT}

The objective of this research was to build an automatable model of process documentation. It is expected that this model allow fulfilling the requirements of several management systems, so that an organization can standardize and automate them, confident that, at the same time, it will comply with the standards established by the Colombian National Accreditation Council (cNA, by its initials in Spanish) and the ISO 9001 standard, as well as Business Process Management (BPM). The analysis of these models was meticulous and comparative and the results were used to complement the contents of the manual. The resulting model was applied directly in the organizational context. The research results show that it is possible to document processes in detail, to comply with international standards, to respond to change rapidly on the basis of improvements in processes and carry over the content of the proposed model to a Business Process Management Suite (BPMs), thus saving on costs, time and other valuable elements.

Keywords: Automation, BPM, documentation, processes, management system.

\section{RESUMO}

Esta pesquisa pretendeu construir um modelo automatizável de documentação de processos. Espera-se que este permita dar resposta aos requisitos de vários sistemas de gestão, de maneira que uma organização possa estandardizá-los e automatizá-los com a confiança de cumprir, ao mesmo tempo, com o estabelecido tanto pelo Conselho Nacional de Acreditação (CNA), como pela norma ISO 9001 e a Business Process Management (BPM). Analisaram-se de maneira minuciosa e comparativa estes modelos. Os resultados utilizaram-se para complementar o conteúdo do manual. O modelo resultante se aplicou diretamente no contexto organizacional. Os resultados mostram que é possível documentar processos ao detalhe, cumprir com standards internacionais, responder com rapidez a mudanças com base nas melhoras que os processos podem apresentar e levar o conteúdo do modelo 
proposto a uma Business Process Management Suite (BPMs), poupando com estes custos, tempo e outros elementos valiosos.

Palavras-chave: automatização, BPM, documentação, processos, sistema de gestão.

\section{INTRODUCCIÓN}

El entorno actual está caracterizado por la globalización y un entorno altamente competitivo para las empresas. Estas se encuentran cada vez más interesadas en alcanzar y demostrar un sólido desempeño operacional mediante las herramientas a su disposición, entre las que se encuentran el control y la gestión de los procesos. Para obtener beneficios y lograr la mayor productividad y rentabilidad posibles deben entender claramente cómo satisfacer al cliente interno y externo.

Algunas organizaciones han concentrado sus esfuerzos alrededor de modelos de gestión que puedan contribuir al logro de la calidad total. Este es un asunto fundamental para poder competir en el entorno actual. Sin embargo, las empresas no siempre enfatizan en la organización de "la casa" y esto hace que los eventos se vean de forma funcional y no transversal. Es en este punto en donde las organizaciones pierden su foco, al ver la gestión de procesos y todo lo que esto conlleva de forma independiente, es decir, sin considerar que su documentación y caracterización representan cadenas de valor que se deben elevar a otros niveles de automatización, seguimiento y mejoramiento continuo.

El presente documento aborda de algún modo esta problemática. Para hacerlo se ha estructurado en tres secciones básicas. En la primera se muestran los resultados de la revisión de la literatura, en la segunda, la metodología utilizada para llevar a cabo la presente investigación y en la tercera, los resultados de la misma. El documento presenta también, para terminar, una serie de conclusiones relevantes que emergen directamente del trabajo realizado.

\section{REVISIÓN DE LA LITERATURA}

Estudios realizados por Gemoets y Mahmood (1990) demostraron la dependencia entre el usuario de un sistema y la existencia de documentación clara respecto al mismo. $\mathrm{Su}$ presencia otorga relevancia, claridad y utilidad para su operación. En efecto, de acuerdo con Doll y Ahmed (1985), "una clara documentación puede tener un buen impacto en la satisfacción del usuario".

Kendall y Losee (1986), en el mismo sentido, sostienen que el uso de una 
buena documentación contribuye al logro de una creciente eficiencia en las operaciones y que esta puede contribuir en la disminución de costos de procesamiento. Algo en lo que también coincide Barbuto (1983) cuando plantea que una buena documentación reduce las dependencias del usuario en la gestión de sistemas de información ("Management Information Systems" -MIS1-. Este conjunto de autores coincide, de un modo u otro, en que la documentación debe presentarse de forma sencilla, clara y concisa, así como en el hecho de que esta debe incorporar la introducción y la manipulación de los sistemas y los datos.

La gestión por procesos puede existir con tecnología o sin ella. Esta consideración sustenta, de hecho, una nueva práctica que se pretende utilizar en la norma ISO 9001:2015. Así, todas las actividades alrededor de la gestión por procesos involucran un mecanismo de análisis para observar, medir, controlar y lograr la optimización, la cual será la que delimite el modo de operación de la organización (Meléndez, 2012). La implementación de la gestión por procesos se ha revelado entonces tal vez como la herramienta de gestión empresarial más efectiva para todo tipo de organización. Según Carraher (2013) esta tiene la capacidad de mejorar la eficiencia y la productivi- dad, de reducir los costos, de minimizar los errores y de proporcionar visibilidad sobre el negocio en el cumplimiento de los objetivos y los procesos.

La gestión de los procesos se apoya en la documentación. Sin que esto haya sido percibido necesariamente por todos, el eje central de los procesos en la actualidad es la tecnología de la información (ті о гт por information technology). Esta garantiza que los procesos empresariales cuenten con las aplicaciones y datos que requieren para funcionar. Cabe recordar que los modelos de negocio de los diversos sectores comerciales son cambiantes. Por esta razón es preciso modificar de manera constante los procesos existentes, ya sea para mejorarlos gradual $\mathrm{u}$ ostensiblemente o para crear unos nuevos. Esto significa que la documentación es cambiante y que las aplicaciones de TI existentes también se deben modificar.

El problema se centra en la adaptación de los procesos frente a la TI, ya que en diversas ocasiones ambos elementos no se encuentran muy bien conectados. De hecho, suele existir una desconexión significativa entre estos. Lo ideal es lograr que la organización se mantenga ágil y competitiva. Es en este punto en donde aparecen importantes mecanismos 
como las Business Process Management Suites (BPMS). ${ }^{1}$ Estas permiten la automatización de los modelos de negocio (procesos) en un mundo cambiante.

Este tipo de TI interconecta directamente las estrategias y los procesos. El objetivo es colaborar con el negocio integrándose con otros sistemas, de acuerdo con las condiciones del mercado. Actualmente, las empresas están migrando cada vez más hacia la aplicación de tecnologías con el fin de buscar eficiencia $\mathrm{y}$ efectividad en sus procesos. Esto sin darse cuenta de que la clave para lo anterior es, como se ha indicado antes, desde el principio, la existencia de una correcta documentación. Frente a este fenómeno emerge la siguiente interrogante básica de investigación:

\section{¿CÓMO AUTOMATIZAR \\ PROCESOS ENFOCADOS A \\ UN SISTEMA DE GESTIÓN A \\ PARTIR DE UN MODELO DE DOCUMENTACIÓN BASADO \\ EN BUSINESS PROCESS \\ MANAGEMENT (BPM)?}

El resultado principal que se presenta en este artículo es un modelo que permite a una organización entender, desarrollar y adoptar una cultura de gestión por procesos. Esto identificando su optimización para adaptarlo a la nueva tecnología BPMS.

Los beneficios que se pueden obtener adoptando BPM como metodología, y aplicabilidad con tecnología se reflejan en particular en la posibilidad de llegar a obtener múltiples innovaciones. De acuerdo con Porras (2007) estas innovaciones:

Son aplicaciones de software para la ejecución de los procesos (BPMS o un ESB bus), para almacenar las reglas del negocio (BRE), para monitorear el desempeño de los procesos BAM (Business Activity Monitoring) y, por último, señalamos la sinergia que existe entre BPM y SOA (Arquitectura Orientada a Servicios TI).

Algunos beneficios según Porras (2007) son:

1. Alineación entre la estrategia y la ejecución: La estrategia en la ejecución se facilita con el BPM.

2. Agilidad: Determina en gran medida el éxito o fracaso de las organizaciones. El BPM permite cambios ágiles y en tiempo real sobre los procesos.

1 Una BPMS "es una herramienta para el diseño, implementación y mejora de una actividad o conjunto de actividades que cumplirán un objetivo organizacional específico". Ver http://searchcio. techtarget.com/definition/Business-process-management-suite-BPMS 
3. Conformidad con los marcos regulatorios existentes: La tecnología BPM incorpora el rastreo de los procesos (BAM). Esta opción colabora con los rastros requeridos por la auditoría y otras áreas de proceso. El BAM permite monitorizar puntualmente cada una de las iteraciones realizadas a los procesos que se llevan a la BPMs. Adicionalmente, permite analizar los costos de cada una de las actividades, tiempos, generación de alertas, alarmas, entre otros indicadores de utilidad en la operación de la institución.

4. Orientación al cliente: Las organizaciones se deben principalmente a sus clientes. Por esta razón siempre es necesario tener en cuenta su opinión.

5. Organización orientada a los procesos: Facilita la tarea de modelar y mejorar los procesos de toda la organización.

6. Estrecha la relación entre TI y el negocio: Los cambios en los procesos se perciben de inmediato, ya que estos se soportan en un sistema (software). La relación entre SOA y BPM es cada vez más fuerte.

7. Estandarización: Una vez que el proceso se ha modelado, simulado y configurado, se ejecuta en una BPMs. Cada vez que un usuario consume un proceso, este se convierte en una práctica están- dar para toda la organización. En este punto cobran vida los manuales de procedimientos de las normas ISO 9000, ya que permiten ser controlados y ejecutados de forma dinámica.

La academia enseña que las organizaciones deberían tener documentados todos sus procesos y registros. Esto con el fin de tener controles internos que les permitan obtener un mejor desempeño y gestión. Es importante identificar que cuando los procesos y registros de una organización se implementan y se ponen en marcha, contribuyen con la construcción y aplicación de indicadores que hablan del desempeño de los colaboradores.

La automatización de procesos parte de su documentación, como se ha indicado, pues si se hace bajo estándares internacionales, la BPMS $^{2}$ hace que el lenguaje, el flujo y la comprensión del ejecutor sean bastante eficientes. Resulta pertinente, en este punto, realizar una breve comparación entre la documentación tradicional y una enfocada en una BPMS (tecnología para automatizar procesos) (ver tabla 1). Teniendo en cuenta que la BPM, la ISO y la automatización buscan fines similares es útil pensar en la integración de estos esquemas en un solo modelo.

2 Business Process Management Suite. 
Tabla 1. Documentación tradicional versus documentación enfocada a BPM

\begin{tabular}{|c|c|}
\hline Documentación actual & $\begin{array}{l}\text { Documentación BPM - } \\
\text { Automatización }\end{array}$ \\
\hline Información dispersa. & Información organizada. \\
\hline $\begin{array}{l}\text { Procesos documentados sin estándares } \\
\text { internacionales. }\end{array}$ & Documentación bajo estándares internacionales. \\
\hline $\begin{array}{l}\text { Los cambios en los documentos y su } \\
\text { implementación son procesos lentos y generan } \\
\text { mayor resistencia. }\end{array}$ & $\begin{array}{l}\text { Se dan cambios cuya implementación es ágil. } \\
\text { Se reacciona de manera más rápida ante los } \\
\text { imprevistos. }\end{array}$ \\
\hline $\begin{array}{l}\text { La integración y comunicación entre procesos } \\
\text { no es clara ni evidente. }\end{array}$ & Integra todos los procesos de forma dinámica. \\
\hline $\begin{array}{l}\text { Los procesos se mantienen en secreto y no se } \\
\text { divulgan en todos los niveles de la organización. }\end{array}$ & $\begin{array}{l}\text { Existe transparencia ante las partes interesadas. } \\
\text { Se conocen y se pueden gestionar las mejoras. } \\
\text { Existe una verdadera gestión del conocimiento } \\
\text { en la organización. }\end{array}$ \\
\hline Exceso de papel. & $\begin{array}{l}\text { Eliminación del uso del papel o ahorro del } \\
\text { mismo. }\end{array}$ \\
\hline Exceso de vistos buenos en los procesos. & $\begin{array}{l}\text { Disminución de vistos buenos. Análisis } \\
\text { de generación de valor por facilidad en el } \\
\text { seguimiento. }\end{array}$ \\
\hline No se obtiene información en tiempo real. & Facilidad en el seguimiento en tiempo real. \\
\hline Se confunden estrategias y procesos. & Se evidencian y se ejecutan los procesos. \\
\hline No se identifican los errores y reprocesos. & Se identifican los errores y los reprocesos. \\
\hline $\begin{array}{l}\text { Dificultad para estandarizar tiempos y generar } \\
\text { alertas o alarmas por retrasos. }\end{array}$ & Se generan alertas y alarmas por retrasos. \\
\hline $\begin{array}{l}\text { No se diferencia el diseñador del consumidor } \\
\text { del proceso. }\end{array}$ & $\begin{array}{l}\text { El diseñador y el ejecutor son claramente } \\
\text { definidos e identificables. }\end{array}$ \\
\hline
\end{tabular}

Fuente: Elaboración propia.

El modelo propuesto en este documento permite a una organización desarrollar el sistema de gestión de calidad, ya que responde a los requisitos de la norma ISO 9001, al entorno cambiante, a la identificación de errores y falencias del proceso y a los estándares internacionales, entre otros aspectos.

\section{METODOLOGÍA}

Esta es una investigación de tipo documental (Glenn, 2009). Se basa, específicamente, en 1) el análisis de manuales de procesos de instituciones de educación superior nacionales e internacionales, 2) la determinación de sus similitudes y diferencias, 
3) la evaluación del cumplimiento de la norma ISO 9001:2008, 4) la realización de un análisis cualitativo para diseñar los niveles de documentación de procesos alineados con la automatización y 5) la construcción del modelo de documentación de procesos automatizables basado en BPM, con la aplicación en un proceso.

\subsection{Desarrollo}

A continuación se analizan las etapas de la investigación, según la metodología descrita.

\subsubsection{Manuales de procesos de entidades de educación superior}

Se realizó un análisis detallado de los manuales de procesos y procedimientos utilizados e implementados por once instituciones reconocidas. Se diseñó un cuadro comparativo para determinar los ítems que se presentan con mayor frecuencia y poder así determinar el modelo estándar para un manual de procesos y procedimientos. Se hizo una matriz que permitiera definir los parámetros que se integraban dentro de los manuales, incluidos aquellos de los procesos. Estos se clasificaron de acuerdo con la experiencia de la autora respecto a su importancia y su potencial de generación de valor. Como conclusión, se obtuvo un contenido inicial para un manual de calidad, la descripción de procesos y los elementos específicos que se deben utilizar en la documentación. Después de realizar el mapeo (matriz), se consignaron en un cuadro los ítems que el modelo estándar debería contener (ver tabla 2 ). 


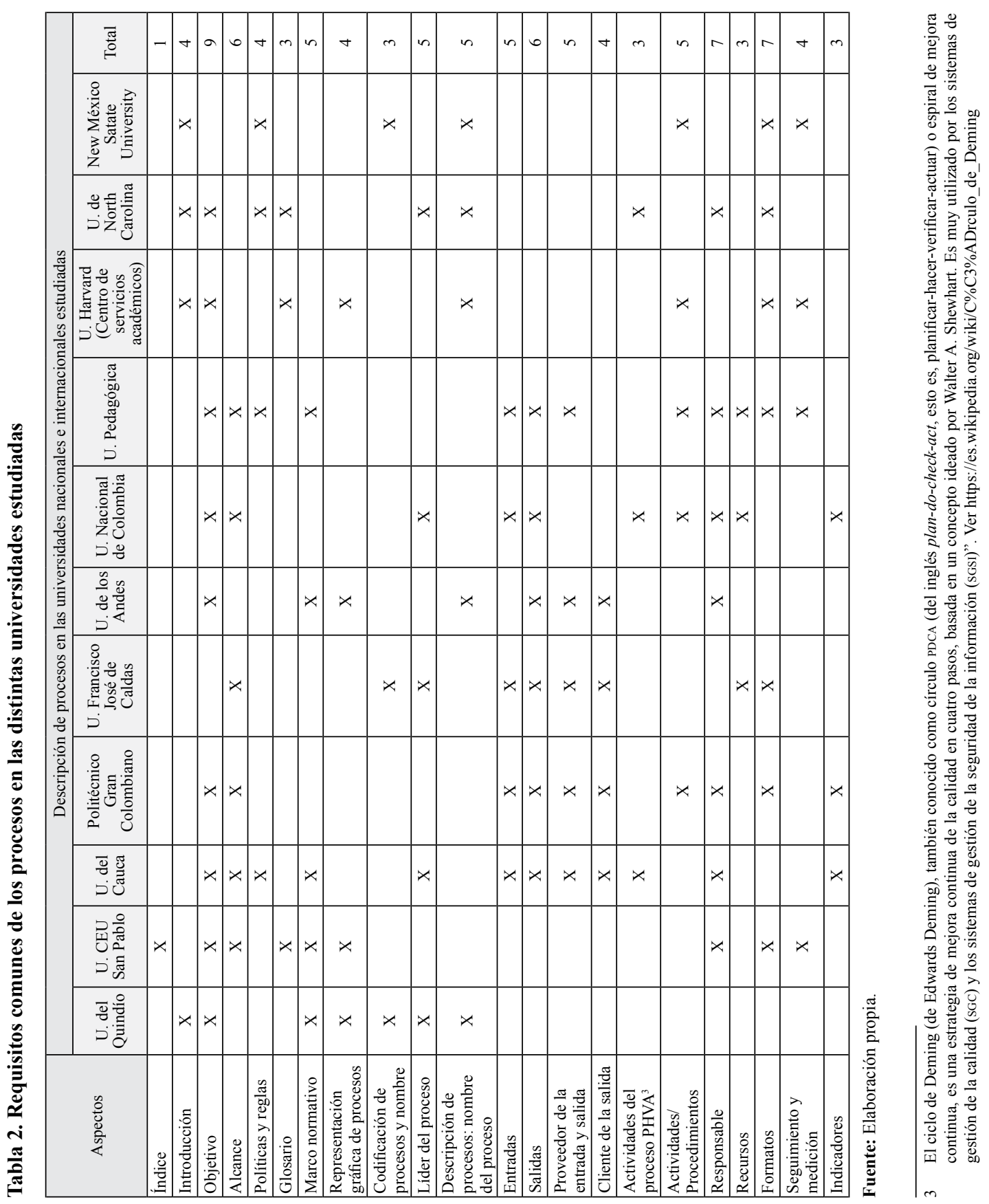


Con base en este análisis, realizado a partir de la literatura estudiada y en particular de los referentes considerados, se concluyó que un manual de procesos estándar debería estar conformado de la siguiente manera:

1. Introducción

2. Objeto

3. Alcance

4. Marco normativo

5. Definiciones

6. Estructura organizacional

7. Mapa de procesos

8. Definición básica del proceso

9. Diagrama de flujo

10. Descripción de los procesos y procedimientos

a. Objetivo del proceso

b. Alcance del proceso

c. Marco normativo específico del proceso

d. Descripción, entradas, salidas, partes interesadas, actividades, responsables, formatos.

e. Anexos

f. Indicadores

g. Control de cambios

11. Responsable de la documentación

2.1.2. Congruencia entre el manual estándar, un sistema de gestión ISO 9001 y sus ocho principios

Con base en un análisis de la norma y los principios se identificaron y definieron los conceptos, requisitos y el sistema de gestión de calidad. Se realizó una matriz que muestra la congruencia de los principios, el sistema de gestión de calidad y el manual de procesos estándar. Como conclusión, los elementos contemplados en el manual de procesos estándar responden a los requisitos del sistema de la norma (ver anexo 1).

\subsubsection{Descripción de los elementos del modelo BPM}

La BPM aporta de manera directa a la administración del negocio. En la actualidad es reconocida como una metodología que permite orientar los esfuerzos de las organizaciones para optimizar los procesos y procurar su eficiencia y eficacia, a través de una gestión sistemática de los mismos. Para esto, los procesos se deben documentar, modelar, automatizar, integrar con sistemas transaccionales, monitorear, publicar y mejorar de forma continua. Luego del análisis realizado en este trabajo se propusieron subdimensiones transversales a las dimensiones de la BPM. Estas se contemplan en la definición del modelo objeto de este trabajo. Ellas son:

- De la estructura organizacional: Permite representar por medio de un diagrama los diferentes niveles de jerarquización. Su detalle 
contribuye a la definición de visto bueno (Vo.Bo.) y a las validaciones de los procesos.

- Del talento humano: Comprende el personal calificado con el nivel y la autoridad requeridos para ejecutar los procesos. Un recurso humano interdisciplinario capaz de trabajar en equipo, conocedor de la misión, las responsabilidades, los medios y las limitaciones de la organización y su operación.

- De los marcos normativos: Son políticas, reglas, lineamientos, valores, manuales y documentos.

- De la infraestructura física y tecnológica: Identificar otros sistemas como Customer Relationship Management (CRM), Enterprise Resource Planning (ERP), gestor documental, entre otros, para la integración de datos en los procesos.

- De la resistencia al cambio: Plantear la manera de reaccionar a los cambios con el fin de disminuir la brecha que se presenta con las nuevas implementaciones de automatización de los procesos, sin que esto suponga un aumento representativo en los costos y los beneficios de la organización. Conviene crear una mesa de ayuda para brindar soporte a los usuarios y disminuir el impacto de la nueva tecnología.

- De la seguridad y control: Considerar puntos de control en la definición y durante la operación de los procesos. Esto para la construcción de indicadores de mejoramiento continuo.

- Del mejoramiento (BAM, análisis de reportes): Cada iteración de proceso puede tener comportamientos diferentes. Esto debido a que el ingreso de datos en el flujo de los procesos no siempre es estándar. La base del mejoramiento radica en el PHVA.

\subsubsection{Modelo documental de aplicaciones de BPM}

Se realizó una investigación de modelos de documentación utilizados en las aplicaciones de BPMS vigentes en el mercado. La búsqueda demostró, entre otras cosas, la falta de información pública. Para el propósito de esta investigación casas matrices de software como Auraportal, Vision Software (Bizagi) y Bonitasoft facilitaron los modelos de documentación de procesos que generan estas herramientas. Se realizó un análisis detallado de los conceptos y criterios utilizados en estos.

Después de realizar el mapeo (matriz) se consignaron en un cuadro los conceptos de cada herramienta. Se hizo un listado que evidencia los parámetros comunes en los manuales de procesos, para definir el modelo que debería utilizar cualquier herramienta BPMS. 


\section{RESULTADOS}

\subsection{Lineamientos para automatizar}

Se estipularon algunos lineamientos que conviene utilizar cuando se piensa en una automatización de procesos. Su uso puede permitir la generación, manejo de datos y documentos electrónicos, algunos con intervención humana y otros automáticos, generados por la BPMS o por la integración de esta con otros sistemas. Dado lo anterior, se considera que los lineamientos para automatizar deben tener presente lo siguiente:

1. La automatización de procesos debe ser adoptada en las organizaciones mediante una estrategia de acceso gradual.

2. Los procesos que se van a automatizar deben ser primero descritos y diagramados, incluyendo todas las actividades, el alcance, los puntos de adopción de las reglas del negocio, entre otros aspectos.

3. Se debe adquirir una BPMS que no requiera programación o generación de código. Esto para que el usuario funcional pueda modelar y ejecutar los procesos.

4. Se debe definir el proceso que se va a implementar.
5. Hay que documentar, modelar y simular el proceso según el modelo de documentación propuesto en este trabajo.

6. Se debe mantener un pensamiento crítico, de eficiencia y efectividad en el proceso con el fin de organizar el modelo apuntando a la generación de valor y optimización del mismo. Lo anterior debe evitar que se automatice el error.

7. Hay que analizar el proceso e identificar en su comportamiento (flujo) las actividades importantes, sobrecargadas operativamente y adecuadas para que las ejecute el sistema y no una persona.

8. Los formularios del proceso solo deben contener la información pertinente y requerida para el análisis o la toma de decisiones. Se debe excluir la información que no aporte a la misión de la actividad.

9. El flujo del proceso debe soportar la confidencialidad, la integridad y el resguardo de la información. Esto de acuerdo con las políticas de la institución.

10. No se debe duplicar información entre las bases de datos de la organización.

11. Hay que garantizar la utilización de una interfaz basada en Web Services $^{4}$ que permita la inter-

4 "Un servicio web (en inglés, Web Service o Web Services) es una tecnología que utiliza un conjunto de protocolos y estándares que sirven para intercambiar datos entre aplicaciones. Distintas 
operabilidad con los sistemas de otras entidades.

12. Se debe facilitar el acceso a la aplicación a través de una clave de usuario y contraseña.

13. La información solo se debe presentar con los privilegios pertinentes a las personas autorizadas para verla, usarla, modificarla o eliminarla.

Los lineamientos de automatización se deben utilizar cuando se documenten los procesos bajo el modelo propuesto en este trabajo.

\subsection{Modelo integrado (principios,} ISO, modelo) versus BPMS

Se realizó la integración del Anexo 1 con el modelo de BPMS (modelo documental de aplicaciones de BPM). La integración de los conceptos arrojó relaciones de igualdad y diferencias. Se analizó cada uno de estos aspectos y algunos se consideraron importantes para la docu- mentación del modelo de automatización de procesos para un sistema de gestión (ver anexo 2).

Se puede visualizar al interior de la tabla aquellos espacios que se identifican de la siguiente forma:

Esta imagen, en los lugares en los que aparece, indica que el concepto del modelo de BPMS es acorde con el del modelo estándar presentado.

\subsection{Modelo de automatización de procesos para un sistema de gestión a partir de un modelo de documentación basado en BPM}

El modelo automatizable de documentación de procesos da respuesta a los requisitos provenientes de varios otros modelos. De esta manera, una organización podrá estandarizarlos y automatizarlos con la confianza de cumplir, al mismo tiempo, con lo establecido por el CNA, la norma ISO 9001 y la teoría de la BPM. Este se presenta en la tabla 3.

aplicaciones de software desarrolladas en lenguajes de programación diferentes, y ejecutadas sobre cualquier plataforma, pueden utilizar los servicios web para intercambiar datos en redes de ordenadores como Internet. La interoperabilidad se consigue mediante la adopción de estándares abiertos" Ver https://es.wikipedia.org/wiki/Servicio web 
Tabla 3. Modelo de automatización de procesos propuesto

\begin{tabular}{|c|c|c|c|c|}
\hline \multicolumn{5}{|l|}{ Introducción o motivación del manual } \\
\hline \multicolumn{5}{|c|}{ Autor del modelo/responsable de la documentación } \\
\hline \multicolumn{5}{|l|}{ Objeto del manual } \\
\hline \multicolumn{5}{|l|}{ Definición de los elementos del BPMN } \\
\hline \multicolumn{5}{|l|}{ Marco normativo } \\
\hline \multicolumn{5}{|l|}{ Definiciones } \\
\hline \multicolumn{5}{|l|}{ Estructura organizacional } \\
\hline \multicolumn{5}{|l|}{ Mapa de procesos } \\
\hline \multicolumn{5}{|c|}{ Clave o código, versión y fecha de generación de documento } \\
\hline \multicolumn{5}{|c|}{ Nombre del proceso } \\
\hline \multicolumn{5}{|l|}{ Responsable o dueño del proceso } \\
\hline \multicolumn{5}{|l|}{ Objetivo del proceso } \\
\hline \multicolumn{5}{|l|}{ Descripción o alcance del proceso } \\
\hline \multicolumn{5}{|l|}{ Marco normativo especifico del proceso } \\
\hline \multicolumn{5}{|l|}{ Recinto seguro } \\
\hline \multicolumn{5}{|c|}{ Descripción de reglas de negocio asociadas al proceso } \\
\hline \multicolumn{3}{|l|}{ Diagrama o modelo en BPMN } & & \\
\hline \multicolumn{2}{|c|}{ Nombre, código y descripción de la operatividad de los eventos } & Eventos & & \\
\hline \multicolumn{2}{|c|}{$\begin{array}{l}\text { Nombre, código y descripción de la operatividad de las tareas } \\
\text { personales, entradas salidas, reglas de negocio (si aplican), for- } \\
\text { matos, responsable/roles o ejecutores }\end{array}$} & \multirow{11}{*}{$\begin{array}{c}\text { Tareas o } \\
\text { actividades }\end{array}$} & & \\
\hline \multicolumn{2}{|l|}{ Tiempo de ejecución de las tareas personales } & & & \\
\hline Nombre del formulario/formatos & \multirow{4}{*}{$\begin{array}{l}\text { Documentos } \\
\text { asociados a } \\
\text { las tareas }\end{array}$} & & & \\
\hline $\begin{array}{l}\text { Descripción del formulario/formato - Nombre } \\
\text { y tipo de campo }\end{array}$ & & & & \\
\hline $\begin{array}{l}\text { Descripción del contenido de información } \\
\text { del campo }\end{array}$ & & & Aplica para & \\
\hline $\begin{array}{l}\text { Documento automático asociado generado en } \\
\text { una tarea personal }\end{array}$ & & & $\begin{array}{c}\text { procesos y } \\
\text { subprocesos o } \\
\text { procedimientos }\end{array}$ & $\begin{array}{l}\text { Elementos } \\
\text { del BPMN }\end{array}$ \\
\hline Preparada o modificada por: & \multirow{3}{*}{$\begin{array}{l}\text { Registro de } \\
\text { modificacio- } \\
\quad \text { nes }\end{array}$} & & & \\
\hline $\begin{array}{l}\text { Fecha y hora de preparación o última } \\
\text { modificación }\end{array}$ & & & & \\
\hline Calendario & & & & \\
\hline \multicolumn{2}{|c|}{$\begin{array}{l}\text { Nombre, código, tipo, tipo de función y descripción de la } \\
\text { operatividad de las tareas de sistema (notificadora, invocadora, } \\
\text { traspasadora, desviadora, ejecutora, entre otras) }\end{array}$} & & & \\
\hline \multicolumn{2}{|c|}{ Documento automático asociado por una tarea de sistema } & & & \\
\hline \multicolumn{2}{|c|}{$\begin{array}{l}\text { Nombre, código, género o tipo y descripción de la operatividad } \\
\text { de las compuertas }\end{array}$} & $\begin{array}{l}\text { Compuertas } \\
\text { o gateways }\end{array}$ & & \\
\hline
\end{tabular}

Fuente: Elaboración propia. 
Por último, se construyó un instructivo para aplicar el modelo propuesto a un proceso piloto. Allí se encuentra la documentación del proceso integrado bajo el modelo de automatización basado en BPM con el modelo del proceso en Business Process Model and Notation (BPMN). Lo anterior se probó automatizando y documentando a través de la herramienta BPMS Auraportal ${ }^{\circledR} .5$

\section{CONCLUSIONES}

- El modelo propuesto permite dar respuesta a los requisitos provenientes de varios otros modelos, con lo cual una organización puede estandarizar y automatizar los procesos cumpliendo con lo establecido por el CNA, la norma ISO 9001 y la teoría vinculada a la BPM.

- El modelo posibilita cumplir con estándares internacionales, responder a cambios ágiles por los hechos y mejoras que los procesos puedan presentar. Este soporta la estrategia convirtiendo la gestión en oportunidades innovadoras y competitivas en el sector en el que se desarrolla la organización.

- Un detallado análisis y documentación del sistema de ges-

5 Ver http://www.auraportal.com/es/productos-bpm-workflow-software\#WHAT tión, enfocado en los procesos que se van a automatizar, es de vital importancia. Es imprescindible desglosarlos al máximo, llegando a un profundo nivel de detalle. Si lo anterior se realiza a un nivel superficial, no será viable la implantación de una BPMS ni la automatización eficiente del proceso.

- Un factor importante para el éxito en la documentación para automatización de procesos es, justamente, la cultura de trabajo mediante procesos. Las habilidades y competencias del talento humano que participa en la operación de estos, cuando son automatizados, constituyen uno de los pilares a la hora de abordar iniciativas de mejoramiento documental.

- La documentación de los procesos bajo el modelo propuesto permite que se pueda llevar a una BPMS. Los resultados garantizan la información necesaria para la automatización, así como la comprobación entre lo modelado y lo descrito. Esto permite optimizar tiempo, costos y otros recursos valiosos. Su estandarización define un lenguaje único para todos los procesos desde el punto de vista integrado (actividades, manuales y automatización).

- Una de las claves del éxito en la documentación de los procesos, 
para automatizarlos, es la identificación de las interfaces (integraciones con otros sistemas), ya que en ellos generalmente se encuentran los mayores esfuerzos para implementar plataformas tecnológicas en los procesos.

- Si las BPMs constituyen software orientado a la automatización de los procesos de las organizaciones, cuyo objetivo final es ayudar a la empresa a obtener mejores resultados económicos mediante una organización adecuada de los flujos de información, mejor control y reducción de tiempos, con mayor razón la documentación de los procesos debe ser acorde a la automatización. Esto con el fin de evitar retrabajos o reprocesos en materia de documentación, para hacer que estos se ajusten al modelo de la BPMS.

- La estandarización del modelo de documentación para la automatización de procesos permite definir un lenguaje único para todos ellos. Esto desde un punto de vista integrado (actividades, manuales y automatización).

- El modelo resultante se aplicó a un proceso específico. Sus resultados muestran que es posible contar con procesos documentados al detalle que permitan entender la lógica y magnitud del negocio. Este cuenta con están- dares internacionales y facilita la identificación de mejoras. La documentación en la máquina de BPMS es sencilla y ágil. Esto significa una reducción de tiempos y costos en la automatización. La experiencia con los ejecutores es amigable, puesto que estos entienden el proceso de inicio y comprenden que cada uno aporta valor.

- Al ejecutar el modelo se evidencia la integración de los procesos, se facilita el seguimiento, se mantiene la gestión del conocimiento en la institución, se logra identificar oportunidades de mejora y se soportan la confidencialidad, la integridad y el resguardo de la información.

- El modelo soporta la estrategia, convirtiendo la gestión en oportunidades innovadoras y competitivas en el sector en el que se desarrolla la organización.

\section{AGRADECIMIENTOS}

La autora agradece la participación y los aportes del M.Sc. Rodrigo Gutiérrez Cabrera y, en general, de la Escuela Colombiana de Ingeniería Julio Garavito.

\section{REFERENCIAS}

Barbuto, P. F. (1983). A structured approach to maintenance. En Ed., 
Auerbach information management series: Computer programming management. Pennsauken: Auerbach.

Carraher, K. (2013). Why /por qué BPM (Business Process Management [Versión para lector digital]. Recuperado de http://issuu. com/bpmteca/docs/ebook_why_ porque_bpm_75c3d2cbb7a072

Doll. W.J., \& Ahmed, M. U., (1985), Documenting information systems for management: A key to maintaining user satisfaction. Information and Management, 8(4), 221-226.

Gemoets, L.A., \& Mahmood, M. A. (1990). Effect of the quality of user documentation on user satisfaction with information systems. Information \& Management, 18(1), 47-54.
Glenn, A. B. (2009). Document analysis as a qualitative research method. Qualitative Research Journal, 9(2), 27-40.

Kendall, K. E., \& Losee, R. D. (1986). Information system folklore: A new technique for system documentation. Information \& Management, 10(2), 103-111. Meléndez Esquivel, A. I. (2012). La definición detrás de BPM. The GBM Journal, 16(54), 1-1. Recuperado el 16 de marzo de 2013, de http://web1.gbm.net/bt/ bt54/

Porras Cedeño, G. (2007). Beneficios del вPM. The GBM Journal, 10(37), 1-1. Recuperado el 15 de abril de 2013, de http:// web1.gbm.net/bt/bt37/opinion/ beneficios_del_bpm.php 
Modelo de automatización de procesos para un sistema de gestión

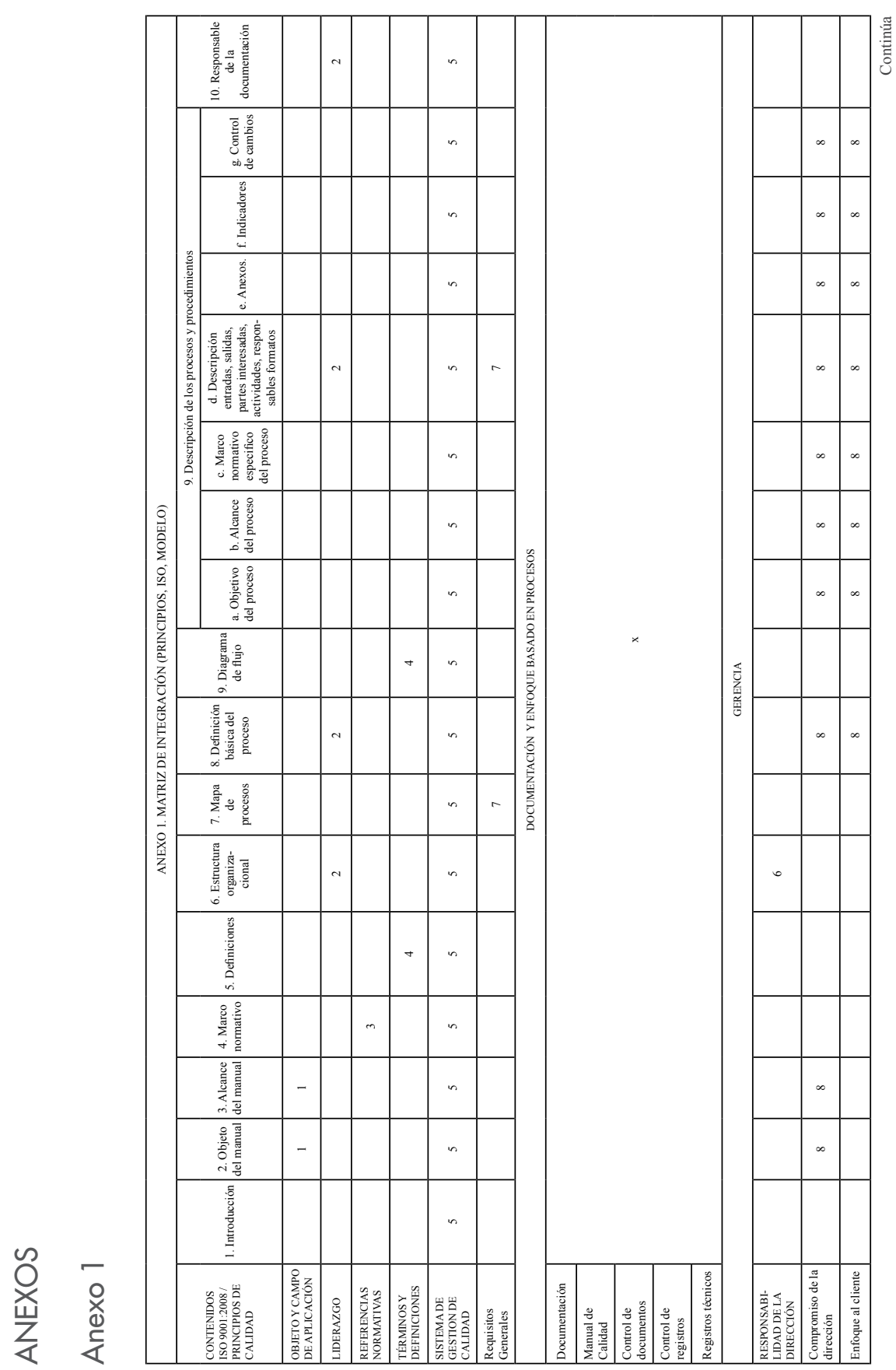

148 Universidad \& Empresa, Bogotá (Colombia) 17129): 131-155, julio-diciembre 2015 
Karen López Supelano

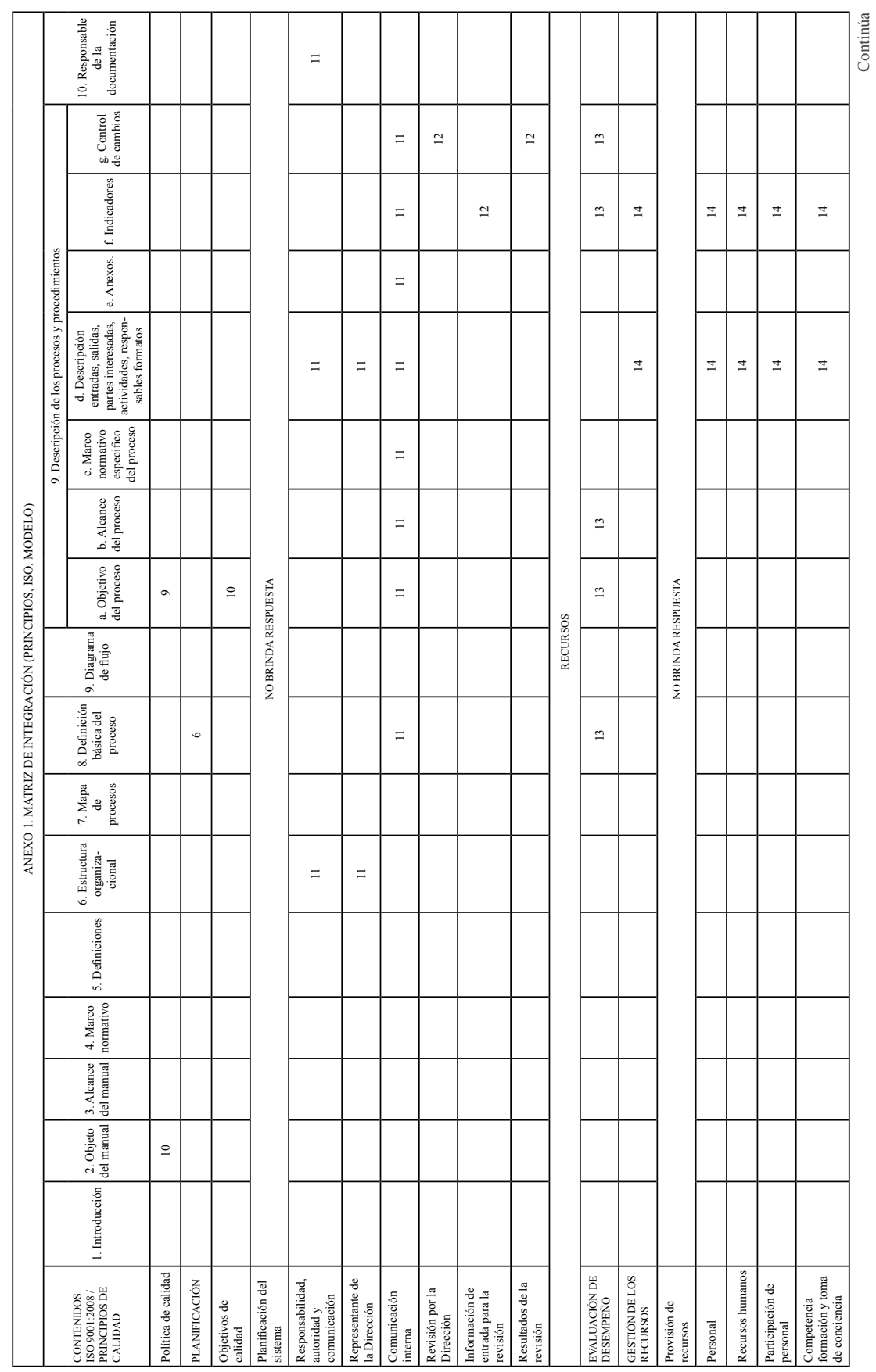




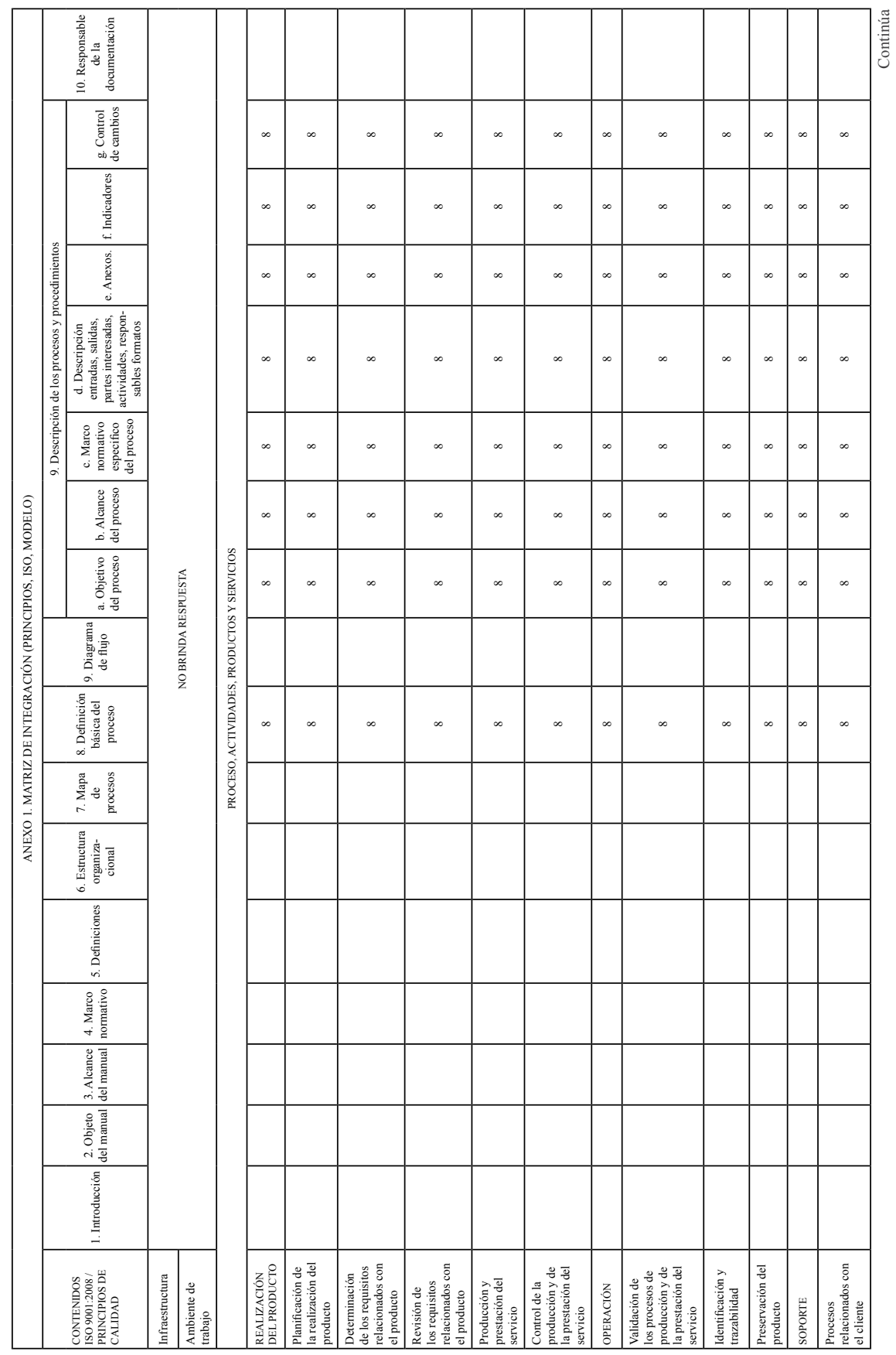


Karen López Supelano

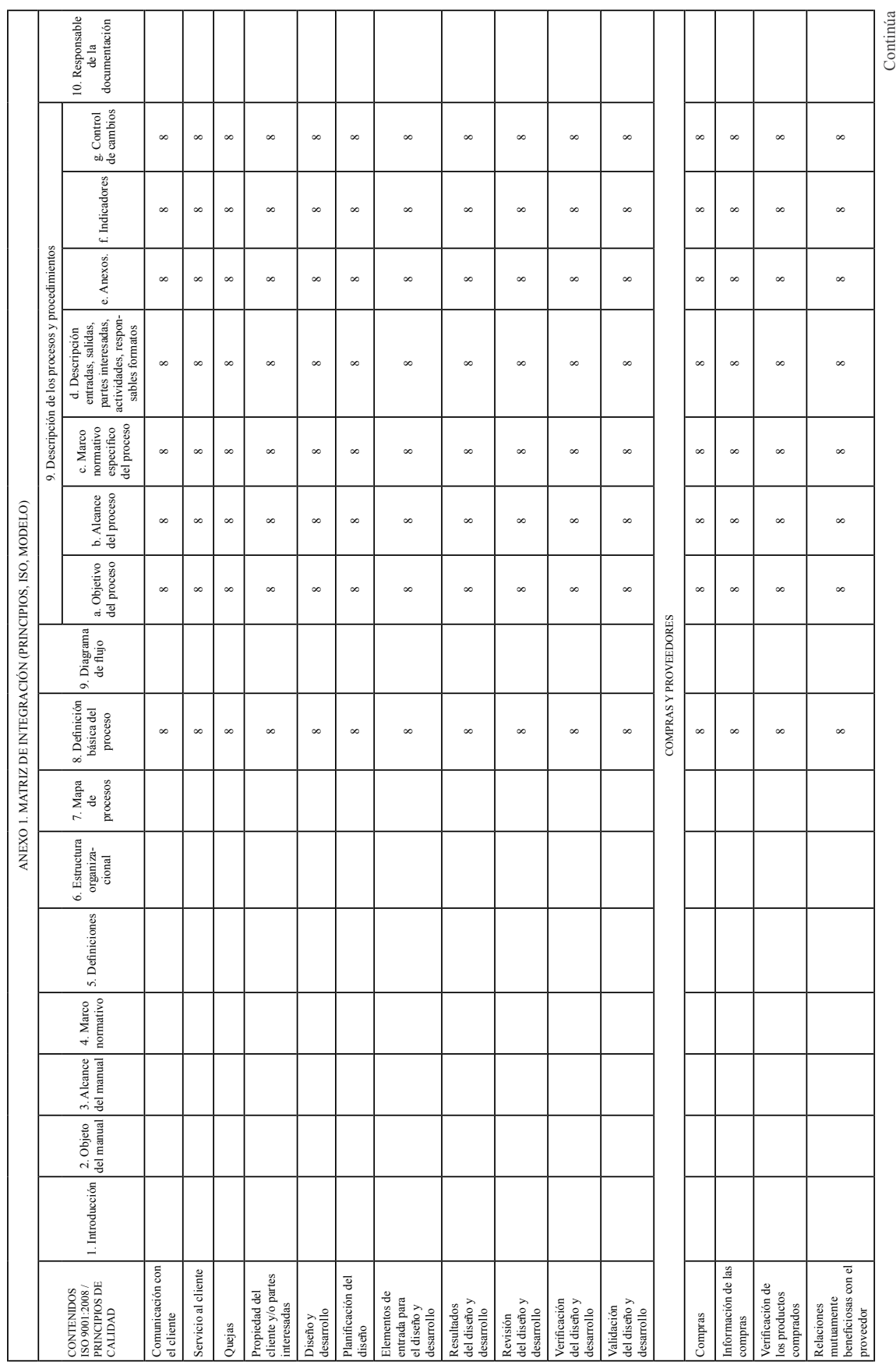




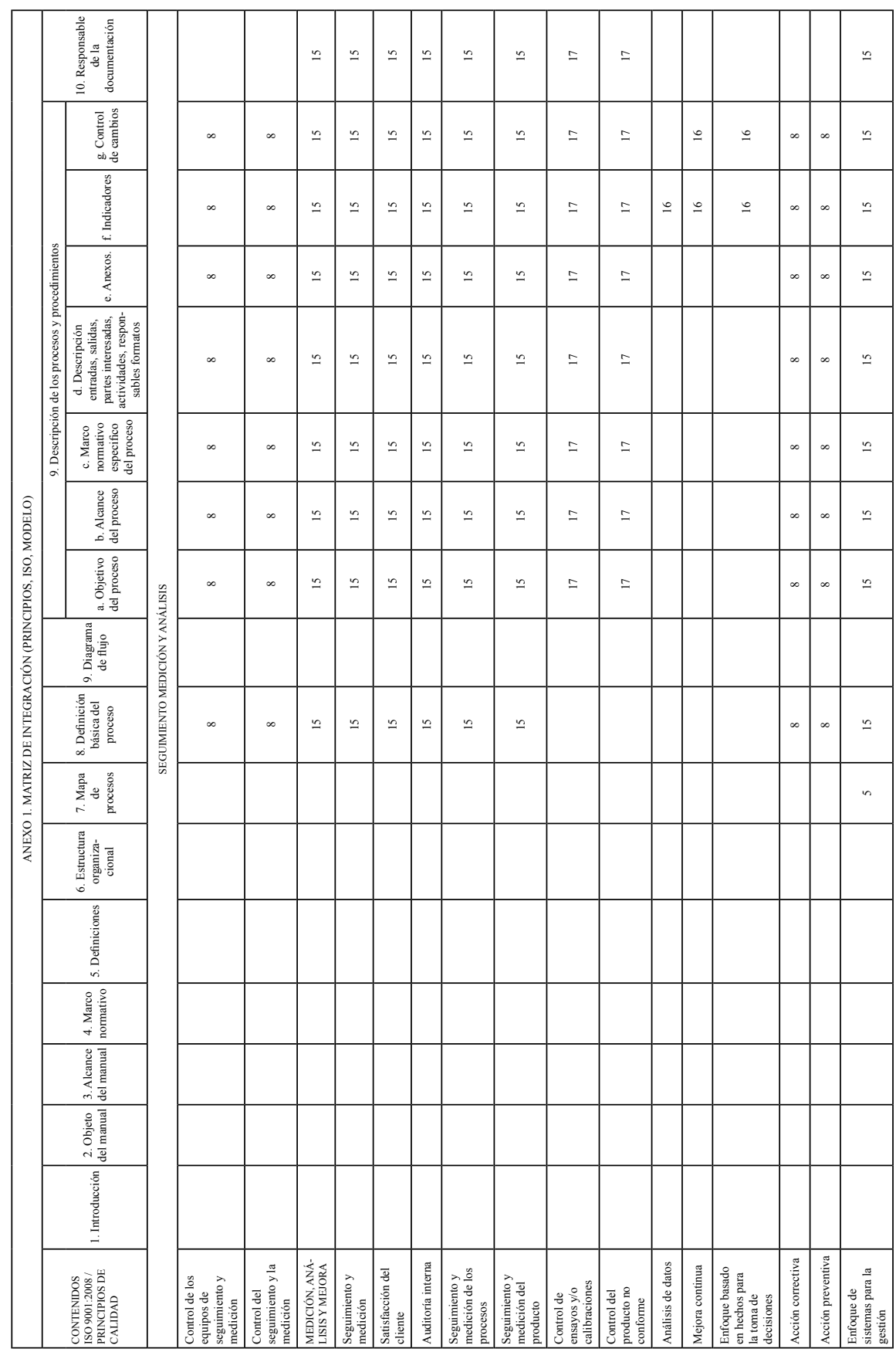


Karen López Supelano

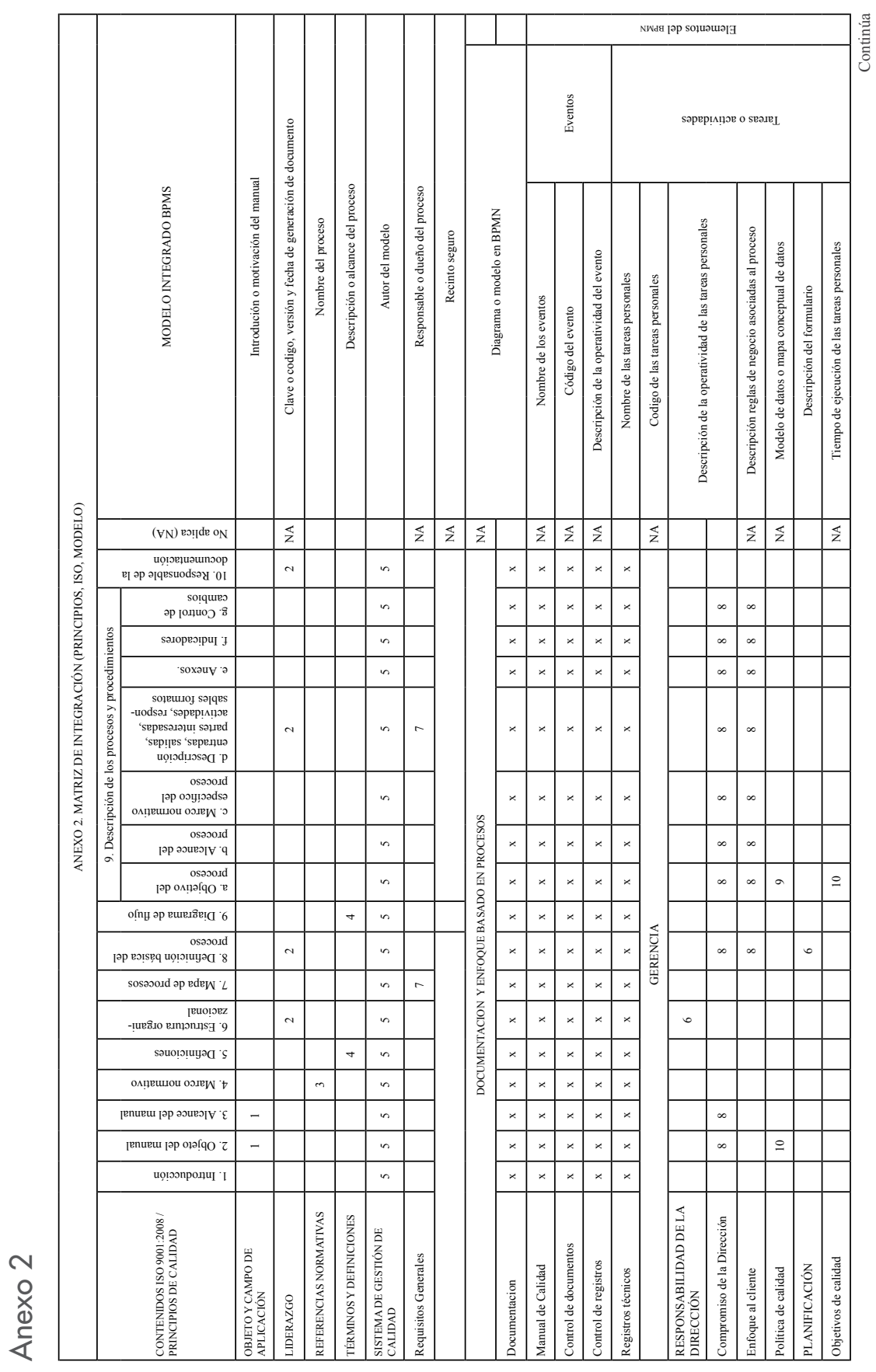


Modelo de automatización de procesos para un sistema de gestión

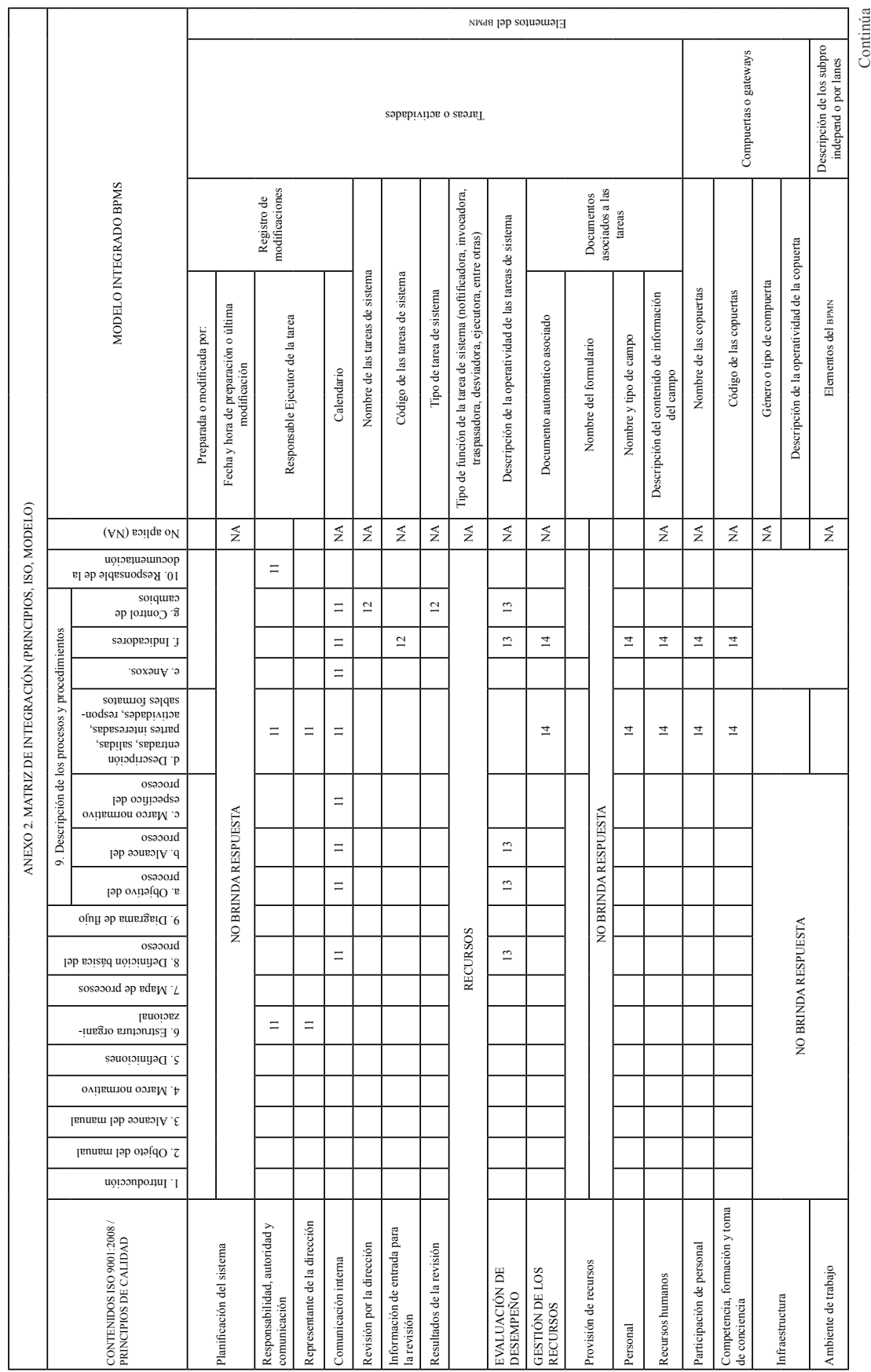

154 Universidad \& Empresa, Bogotá (Colombia) 17129): 131 1-155, julio-diciembre 2015 


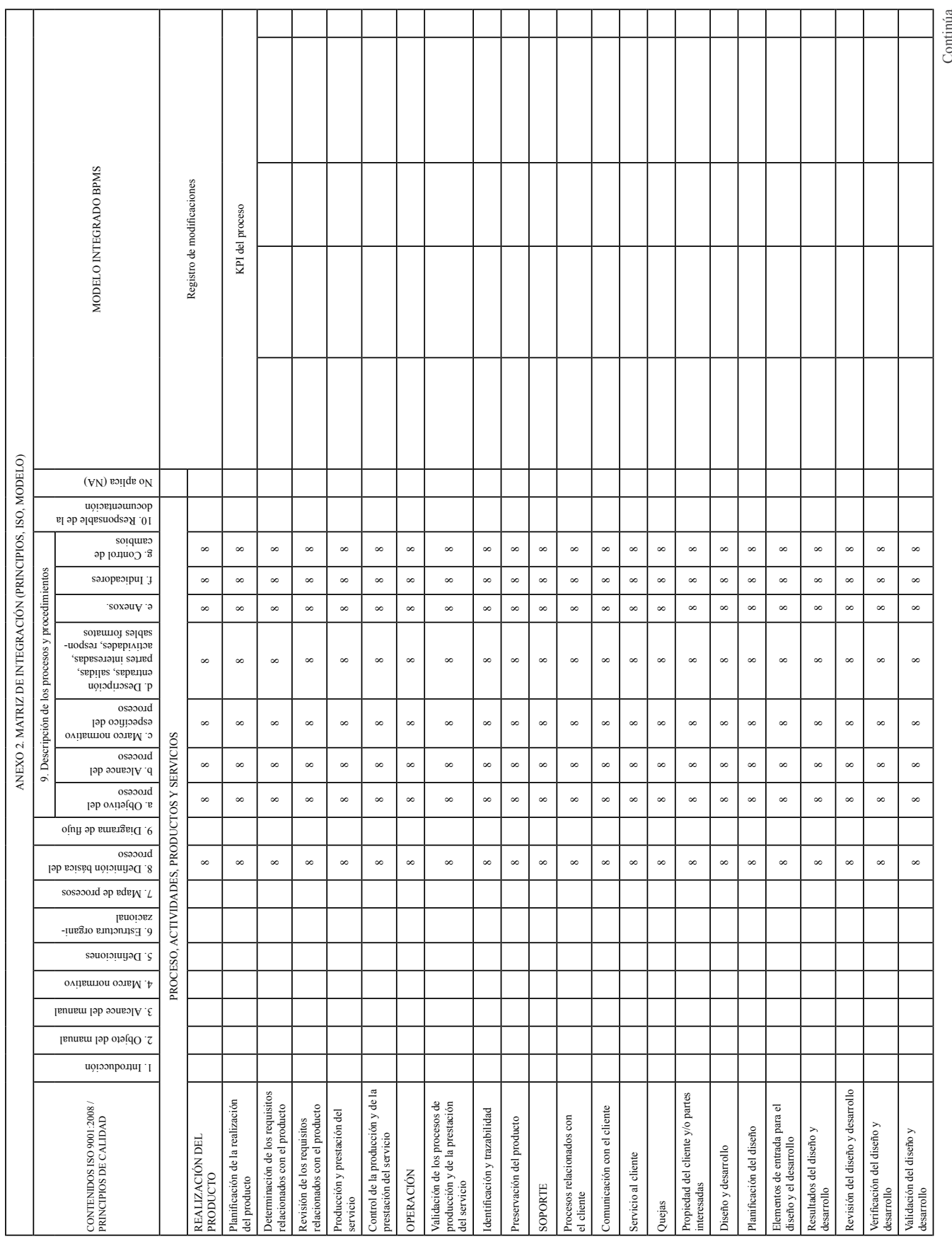




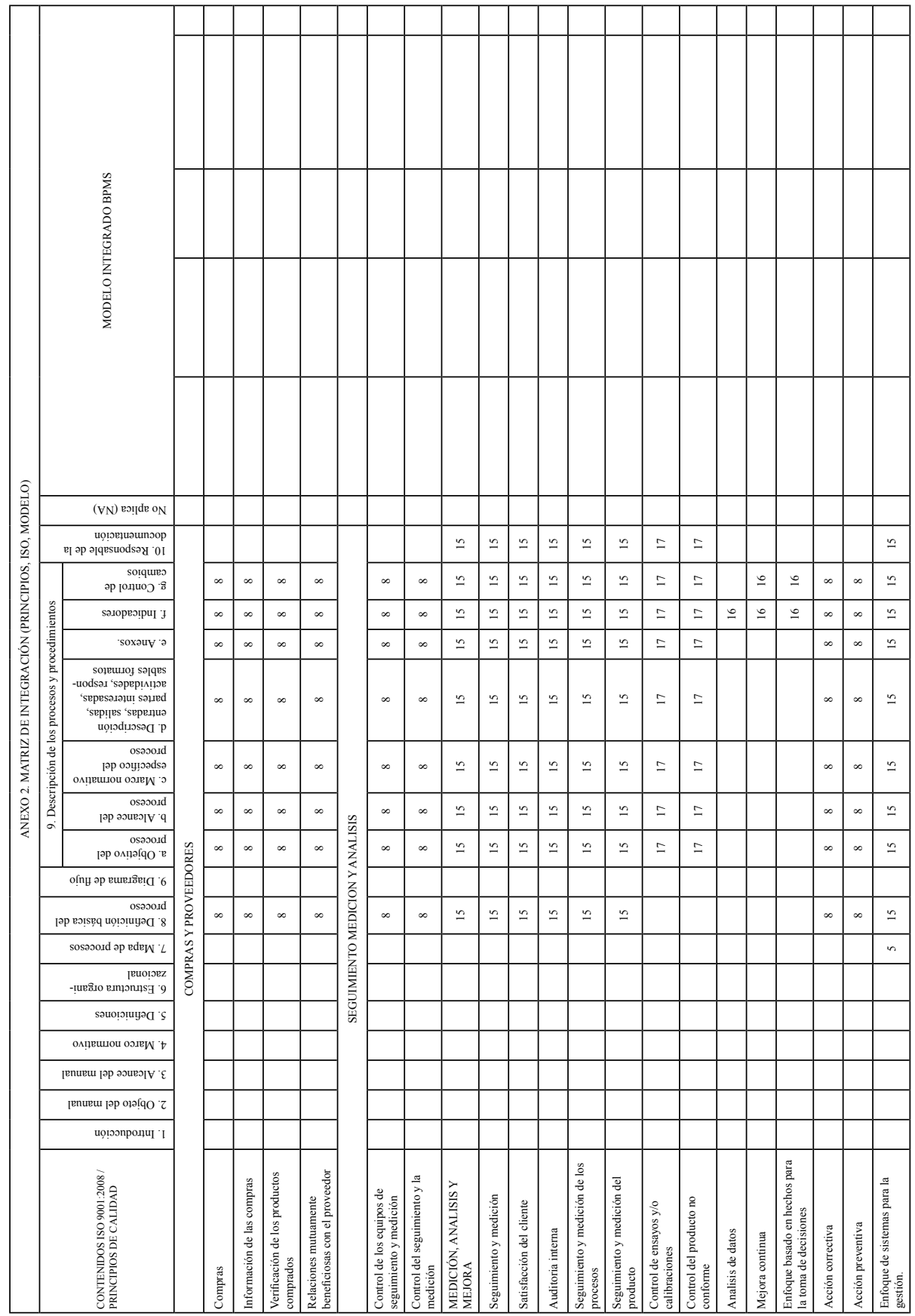

